\title{
Role of microRNA-375-3p-mediated regulation in tinnitus development
}

\author{
KYU-HEE HAN ${ }^{1}$, HYEEUN CHO ${ }^{2}$, KYEO-RYE HAN $^{2}$, SEOG-KYUN MUN ${ }^{2}$, \\ YOUNG-KOOK KIM ${ }^{3}$, ILYONG PARK ${ }^{4}$ and MUNYOUNG CHANG ${ }^{2,5}$
}

\author{
${ }^{1}$ Department of Otorhinolaryngology-Head and Neck Surgery, National Medical Center, Seoul 04564; \\ ${ }^{2}$ Department of Otorhinolaryngology-Head and Neck Surgery, Chung-Ang University College of Medicine, Seoul 06974; \\ ${ }^{3}$ Department of Biochemistry, Chonnam National University Medical School, Hwasun, Jeollanam-do 58128; \\ ${ }^{4}$ Department of Biomedical Engineering, Dankook University College of Medicine, Cheonan, \\ Chungcheongnam-do 16890; ${ }^{5}$ Department of Otorhinolaryngology-Head and Neck Surgery, \\ Chung-Ang University Hospital, Seoul 06973, Republic of Korea
}

Received March 22, 2021; Accepted May 11, 2021

DOI: $10.3892 / \mathrm{ijmm} .2021 .4969$

\begin{abstract}
Changes in the dorsal cochlear nucleus (DCN) following exposure to noise play an important role in the development of tinnitus. As the development of several diseases is known to be associated with microRNAs (miRNAs/miRs), the aim of the present study was to identify the miRNAs that may be implicated in pathogenic changes in the DCN, resulting in tinnitus. A previously developed tinnitus animal model was used for this study. The study consisted of four stages, including identification of candidate miRNAs involved in tinnitus development using miRNA microarray analysis, validation of miRNA expression using reverse transcription-quantitative PCR (RT-qPCR), evaluation of the effects of candidate miRNA overexpression on tinnitus development through injection of a candidate miRNA mimic or mimic negative control, and target prediction of candidate miRNAs using mRNA microarray analysis and western blotting. The miRNA microarray and RT-qPCR analyses revealed that miR-375-3p expression was significantly reduced in the tinnitus group compared with that in the non-tinnitus group. Additionally, miR-375-3p overexpression via injection of miR-375-3p mimic reduced the proportion of animals with persistent tinnitus. Based on mRNA microarray and western blot analyses, connective tissue growth factor $(C T G F)$ was identified as a potential target for miR-375-3p. Thus, it was inferred that CTGF downregulation by miR-375-3p may weaken with the decrease in miRNA expression, and the
\end{abstract}

Correspondence to: Dr Munyoung Chang, Department of Otorhinolaryngology-Head and Neck Surgery, Chung-Ang University College of Medicine, 84 Heukseok-ro, Dongjak-gu, Seoul 06974, Republic of Korea

E-mail: cadu01@cau.ac.kr

Key words: microRNA, tinnitus, dorsal cochlear nucleus, apoptosis, axonal sprouting increased pro-apoptotic activity of CTGF may result in more severe neuronal damage, contributing to tinnitus development. These findings are expected to contribute significantly to the development of a novel therapeutic approach to tinnitus, thereby bringing about a significant breakthrough in the treatment of this potentially debilitating condition.

\section{Introduction}

Tinnitus, a condition that affects $7-25 \%$ of the population worldwide, is a phenomenon in which sound is perceived in the absence of sound stimuli (1-4). In 1-2\% of the total population, tinnitus may be associated with debilitating conditions, including insomnia, anxiety, depression, cognitive dysfunction and stress (1-6). Although various approaches, such as medical therapy, dietary supplements, transcranial magnetic/electrical stimulation and sound therapy, have been devised for the treatment of tinnitus, no treatment to date has been reported to produce a clear therapeutic effect $(7,8)$. Therefore, it is crucial to develop new treatment methods for this condition.

A lack of clarity on the mechanisms underlying the development of tinnitus makes it difficult to design an effective treatment strategy. Therefore, the elucidation of the underlying mechanisms will contribute significantly towards identifying a cure for tinnitus. Maladaptive auditory-somatosensory plasticity in the dorsal cochlear nucleus (DCN) after hearing loss has been suggested as one of the mechanisms promoting the development of tinnitus (9-11). Using a temporary threshold shift (TTS) model, our previous study demonstrated that the changes occurring in the DCN following exposure to noise may play an important role in the development of tinnitus and a decrease in auditory projections and subsequent increase in non-auditory projections via axonal sprouting may be important phenomena associated with the occurrence of tinnitus (12). Therefore, the identification of an appropriate checkpoint for these processes may facilitate the development of a treatment strategy for this condition.

MicroRNAs (miRNAs/miRs) are small non-coding RNAs that regulate gene expression via translational inhibition or 
mRNA degradation (13). As the expression of most genes is under miRNA control, the majority of biological processes are regulated by miRNAs to a certain extent. Therefore, disease development is also hypothesized to be closely associated with the activities of miRNAs. Indeed, the pathogenesis of diabetes mellitus, cancer, and cardiovascular and neurological diseases has been reported to be associated with various miRNAs, whereas certain miRNAs were recently recognized as therapeutic targets (14-16). In particular, therapeutic strategies targeting miRNAs have achieved promising results against hepatitis $\mathrm{C}$ viral infection (16).

Accordingly, the present study was undertaken to identify miRNAs that may be implicated in the pathogenesis of tinnitus. miRNA levels were compared in animal models with and without tinnitus following induction of TTS using microarray analysis and reverse transcription-quantitative PCR (RT-qPCR). Additionally, the candidate miRNAs were overexpressed to examine the differences in the expression of their candidate targets. Specifically, the expression levels of miR-375-3p in the DCNs of animals with tinnitus were measured, and the role of miR-375-3p in tinnitus and the involvement of connective tissue growth factor $(C T G F)$ were investigated, in the hope that the identification and targeting of putative miRNAs involved in the pathogenesis of tinnitus may contribute to the development of novel approaches to the treatment of this condition.

\section{Materials and methods}

Animals. The present study was approved by the Institutional Animal Care and Use Committee of Chung-Ang University (2016-00092). All experiments were performed in accordance with the National Institutes of Health Guide for the Care and Use of Laboratory Animals (17). All animals were allowed to acclimate to the laboratory conditions for 1 week prior to the start of the experiments. The animals were housed in a temperature- and humidity-controlled room with a 12-h light/dark cycle, with food and water available ad libitum. The experiments were conducted on 12-week-old male Sprague-Dawley rats. The auditory brainstem response (ABR) recordings, noise exposure and surgical procedures were performed under anesthesia induced by intraperitoneal administration of Zoletil (40 mg/kg, Zoletil 50 ${ }^{\circledR}$; Virbac) mixed with xylazine (10 mg/kg, Rompun ${ }^{\circledR}$; Bayer-Korea, Ltd.).

Experimental design. A total of 102 rats were used in the present study. The study was conducted in four stages (Fig. 1) as follows: i) Identification of candidate miRNAs involved in the development of tinnitus, using microarray analysis; ii) validation of miRNA expression using RT-qPCR; iii) evaluation of the effects of overexpression of the candidate miRNAs on tinnitus; and iv) target prediction of the candidate miRNAs. In each stage, the ABR and gap pre-pulse inhibition of acoustic startle reflex (GPIAS) were recorded in all animals prior to noise exposure, to confirm that none of the animals had hearing loss or tinnitus. All rats were exposed to $6-8 \mathrm{kHz}$ narrow-band noise at $110 \mathrm{~dB}$ sound pressure level (SPL) for $2 \mathrm{~h}$, with the left ear plugged and sutured. This noise-exposure protocol was developed in our previous study and confirmed to induce a TTS on the right side only (12). To detect the hearing changes, the ABR was recorded on day 1, and at 1 and 3 weeks post-noise exposure, whereas GPIAS responses were recorded at 1 and 3 weeks following exposure. The development of tinnitus was determined based on the recorded GPIAS responses.

A total of 8 rats were used in the first stage of the experiment. At 3 weeks post-noise exposure, a total of 5 rats exhibited evidence of tinnitus. Subsequently, 3 rats were randomly selected each from the tinnitus $(n=5)$ and non-tinnitus $(n=3)$ groups. The right DCNs were harvested from these rats, following the protocol outlined in the atlas of Paxinos and Watson (18). The samples were subjected to miRNA microarray analysis. Based on the results of microarray analysis, candidate miRNAs were selected. For the second stage of the experiment, 13 rats were used. The right DCNs were harvested from these rats at 3 weeks following noise exposure. It was observed that 7 rats exhibited evidence of tinnitus at this stage. To validate the candidate miRNAs, RT-qPCR was performed on these samples. The results were analyzed and the miRNAs that exhibited significant differences between the tinnitus and non-tinnitus groups were identified. In the third stage of the experiment, 81 rats were exposed to noise, of which 59 displayed evidence of tinnitus at 1 week post-exposure. To evaluate the role of the candidate miRNAs selected with RT-qPCR and miRNA microarray analyses, a candidate miRNA oligomer (mimic or mimic negative control) was administered into the lateral ventricles of the 59 rats that manifested evidence of tinnitus. At 3 weeks following noise exposure, whether the tinnitus persisted was determined using the GPIAS recordings. In the next stage of the experiment, rats receiving the candidate miRNA oligomer were divided into the following four experimental groups based on the type of oligomer administered and the persistence of tinnitus at 3 weeks following noise exposure: i) Mimic tinnitus, ii) mimic non-tinnitus, iii) control tinnitus and iv) control non-tinnitus groups. In the last stage of the experiment, target prediction analysis for the candidate miRNAs was performed. Three rats were randomly selected from each of the mimic non-tinnitus and control tinnitus groups. mRNA microarray analysis was performed using the three DCN samples obtained from each group. Based on the results of microarray analysis and the analysis performed using the TargetScan miRNA target prediction server (http://www.targetscan.org/cgi-bin/targetscan/vert_72/ targetscan.$c g i$ species $=$ Rat $\&$ gid $=\&$ mir_sc $=$ miR-375-3p\&mir_ $\mathrm{c}=\&$ mir_nc $=\&$ sortType $=$ cs\&allTxs $=\&$ incl_nc $=$ All), all the potential target genes of the candidate miRNA were identified. Subsequently, the expression levels of these candidate target genes were compared between the two experimental groups using western blotting.

$A B R$ recordings. ABR recordings were performed as described previously (19). ABR was measured using SmartEP (version 2.33; Intelligent Hearing Systems) and high-frequency transducers (HFT9911-20-0035). The ABR signals detected between the subcutaneous electrodes at the nape of the neck and the ipsilateral mastoid process were recorded using the contralateral mastoid process as the return. Tone-pip stimuli of 7, 11 and $15 \mathrm{kHz}$ (duration, $5 \mathrm{msec}$; cos shaping, 21 Hz) were delivered in decreasing steps of $5 \mathrm{~dB}$ SPL. The responses were band pass-filtered (100-1,500 Hz), amplified (x100,000) and averaged over 512 stimuli repetitions at each frequency and sound level. The lowest stimulus intensity that induced a 
detectable response was considered as the threshold, and it was assessed by two researchers.

Behavioral test for tinnitus. GPIAS recordings were performed as described previously (12). The recording system used in the present study consisted of a mesh cage with an accelerometer (LIS344ALH; STMicroelectronics), an audio amplifier (PM-5004; Marantz), a full-range loud speaker (TC9FSD13; Vifa/Peerless, Tymphany), a reference microphone, data acquisition hardware (NI DAQ-6341; National Instruments Corporation), a custom-made anechoic noise box, and the LabVIEW (version 2015; National Instrument)-based custom GUI 3.0 software. This LabVIEW-based software was used for acoustic stimulation, startle response acquisition and response analyses.

Acoustic stimulation was carried out using sound waves of $2 \mathrm{kHz}$ bandwidth and $60 \mathrm{~dB}$ SPL; the center frequencies of 7 , 11 and $15 \mathrm{kHz}$ were used as the background noise. A broadband noise burst of $105 \mathrm{~dB}$ SPL for a duration of $50 \mathrm{msec}$ served as the startle stimulus. During each session, 15 gap-conditioned stimuli and 15 non-gap-conditioned stimuli were presented in a random pair order. The gap pre-pulse that occurred in each gap-conditioned stimulus was presented $100 \mathrm{msec}$ before the onset of the startle stimulus and lasted for $50 \mathrm{msec}$. The time interval between the presentation of acoustic stimulations was altered randomly between 17 and $23 \mathrm{sec}$. The gap-conditioned response/non-gap-conditioned response $(\mathrm{G} / \mathrm{N})$ ratios were calculated according to the following equation:

$$
G / N \text { ratio }=\frac{\text { RMS }-\mathrm{GSR}}{\mathrm{RMS}-\mathrm{NGSR}}
$$

where RMS-GSR and RMS-NGSR are the root-mean-squared (RMS) values of the gap-conditioned startle responses (GSR) and the non-GSR (NGSR), respectively. The outliers among the measured startle responses were removed using Grubb's test (20). The GSR and the NGSR were compared using the Mann-Whitney U test. The animals were considered to have no tinnitus if there were significant differences at all frequencies $(\mathrm{P}<0.05)$; otherwise, the rats were considered to have tinnitus.

miRNA microarray analysis. Microarray analysis of the miRNAs was performed at BioCore Co., Ltd. using the Affymetrix miRNA 4.0 microarray (Affymetrix; Thermo Fisher Scientific, Inc.), which contained all the miRNAs in the miRBase Release 20 database (http://www.mirbase. org/), including 30,434 mature miRNA probe sets. RNA was prepared as described previously (21). Briefly, total RNA was extracted from the DCN samples using the TRI Reagent ${ }^{\circledR}$ (MRC). The quality and quantity of the RNA were assessed using an Agilent 2100 BioAnalyzer (Agilent Technologies, Inc.) and a NanoDrop ${ }^{\mathrm{TM}} 1000$ Spectrophotometer (Thermo Fisher Scientific, Inc.), respectively. The RNA was labeled using the FlashTag Biotin RNA Labeling kit (Affymetrix; Thermo Fisher Scientific, Inc.), and the labeled samples were hybridized to GeneChip miRNA 4.0 microarrays (Affymetrix; Thermo Fisher Scientific, Inc.). A hybridization mixture consisting of control oligo B2, 20X hybridization controls (bioB, bioC, bioD and cre), $27.5 \%$ formamide, DMSO, $2 \mathrm{X}$ hybridization buffer and water, was applied to all the samples. Hybridization was performed in an Affymetrix GeneChip Hybridization Oven
640 at $48^{\circ} \mathrm{C}$ and $60 \mathrm{rpm}$ for $16 \mathrm{~h}$. Subsequently, the arrays were stained with stain cocktails (1 and 2) included in the kit, and washed in Affymetrix GeneChip Fluidics Station 450 , in accordance with the FS450_0002 fluidics protocol. Following scanning using an Affymetrix GeneChip Scanner 3000, all the arrays were analyzed using the Transcriptome Analysis Console ${ }^{\mathrm{TM}} 4.0$ software (Applied Biosystems; Thermo Fisher Scientific, Inc.). The CEL files generated were imported into the Gene Expression Workflow in GeneSpring GX, version 14.9.1 (Agilent Technologies, Inc.). Background correction, $\log _{2}$ transformation and probe set summarizing were achieved using the default settings in the GeneSpring software. Subsequently, principal component analysis was performed using a covariance dispersion matrix for data quality control. Unpaired t-tests were performed to compare the individual gene expression data of the noise-exposed tinnitus group with that of the non-tinnitus group.

$R T-q P C R$. Total RNA was extracted from the DCN samples using the QIAzol Lysis Reagent (Qiagen $\mathrm{GmbH}$ ) according to the manufacturer's instructions. The RNA concentration of the samples was determined using a NanoDrop ${ }^{\mathrm{TM}}$ spectrophotometer (Thermo Fisher Scientific, Inc.). cDNA synthesis was performed using the miScript II RT kit (Qiagen $\mathrm{GmbH}$ ), and $\mathrm{qPCR}$ was performed with primers for miR-15b-3p (cat. no. YP00205898), -105 (cat. no. YP00205105), -221-3p (cat. no. YP00204532), -375-3p (cat. no. YP00204362), -455-5p (cat. no. YP00204363), -544-5p (cat. no. YP02116293), -708-5p (cat. no. YP00204490) and -759 (cat. no. YP00206000; all from Qiagen $\mathrm{GmbH}$ ) in a Bio-Rad CFX 96 real-time system (Bio-Rad Laboratories, Inc.) using the miScript SYBR Green PCR kit (Qiagen $\mathrm{GmbH}$ ) as follows: Initial heat activation at $95^{\circ} \mathrm{C}$ for $2 \mathrm{~min}$, followed by 40 cycles of $95^{\circ} \mathrm{C}$ for $10 \mathrm{sec}$ and $56^{\circ} \mathrm{C}$ for $1 \mathrm{~min}$. All PCR reactions were performed under standard PCR conditions; U6 (cat. no. YP00203907; Qiagen $\mathrm{GmbH}$ ) was used as the endogenous control. The relative quantification (RQ) values were calculated from the quantification cycle $(\mathrm{Cq})$ values using the $2^{-\Delta \Delta \mathrm{Cq}}$ method (22).

Validation study using candidate miRNA oligomers. Based on the results of miRNA microarray and RT-qPCR analyses, miR-375-3p was selected as the candidate miRNA. To evaluate its role, miR-375-3p mimic (5'-UUUGUUCGUUCGGCUCGCGUGA-3') (Qiagen GmbH) and $\mathrm{miR}-375-3 \mathrm{p}$ mimic negative control (Qiagen $\mathrm{GmbH}$ ) were administered to 31 and 28 rats, respectively, that exhibited evidence of tinnitus at 1 week post-noise exposure. Once the rats were anesthetized using the method previously described, and placed in a stereotaxic frame, $5 \mu 1$ of miRNA oligomer $(66.67 \mu \mathrm{M})$ was mixed with $12.5 \mu \mathrm{l}$ of Lipofectamine ${ }^{\circledR} 2000$ (Invitrogen; Thermo Fisher Scientific, Inc.) and injected into the right lateral ventricle $(0.8 \mathrm{~mm}$ posterior to the bregma, $1.4 \mathrm{~mm}$ right lateral to the midline, to a depth of $3.4 \mathrm{~mm}$ from the surface of the skull) at an infusion rate of $1 \mu \mathrm{l} / \mathrm{min}$. The speed of needle insertion and withdrawal was maintained at $1.5 \mathrm{~mm} / \mathrm{min}$. The site of craniotomy was closed using bone wax and the scalp was sutured. Two weeks later (i.e., 3 weeks after noise exposure), the GPIAS responses were measured to determine whether tinnitus persisted. The rats were divided into four experimental groups based on the type of oligomer 


\begin{tabular}{|c|l|l|l|l|c|}
\hline I day & Noise \\
for TTS
\end{tabular}

Figure 1. Experimental design. TTS, temporary threshold shift; GPIAS, gap pre-pulse inhibition of acoustic startle reflex; ABR, auditory brainstem response; RT-qPCR, reverse transcription-quantitative PCR.

administered and the persistence of tinnitus, as follows: i) Mimic tinnitus, ii) mimic non-tinnitus, iii) control tinnitus, and iv) control non-tinnitus groups.

Target prediction of the candidate miRNA. To identify the target mRNA regulated by miR-375-3p, mRNA microarray analysis was performed on the DCN samples from 3 rats randomly selected each from the mimic non-tinnitus and control tinnitus groups. Microarray analysis of the mRNAs was performed at BioCore Co., Ltd. Briefly, RNA was isolated and prepared as detailed above. The cDNAs were generated using the GeneChip Whole Transcript PLUS Reagent kit (Affymetrix; Thermo Fisher Scientific, Inc.) and labeled with terminal deoxynucleotidyl transferase (TDT) using the Affymetrix proprietary DNA Labeling Reagent (Affymetrix; Thermo Fisher Scientific, Inc.). The labeled samples were hybridized to the GeneChip RaGene 2.0 ST Array (Affymetrix; Thermo Fisher Scientific, Inc.). All arrays were scanned using the Affymetrix GeneChip Scanner 3000, and raw analysis was performed using the Transcriptome Analysis Console ${ }^{\mathrm{TM}}$ software. The CEL files generated were imported into the Gene Expression Workflow in GeneSpring GX version 14.9.1 (Agilent Technologies, Inc.). Subsequently, the microarray data were analyzed as described above.

Western blot analysis. Western blotting was performed to detect the expression levels of the candidate target genes obtained using mRNA microarray and miRNA target prediction server (TargetScan) analyses. In addition, western blotting was performed to determine the expression levels of the candidate target genes along with $C T G F$, which is a previously reported target of miRNA-375-3p (23). The expression levels of the proteins were compared between the mimic non-tinnitus and control tinnitus experimental groups.

Western blotting was carried out on the DCNs, as described previously (12). Briefly, the homogenized DCN samples were treated with RIPA lysis buffer (Biosesang) and a 100X Protease Inhibitor Cocktail (EDTA-free, lyophilized; cat. no. QTPPI1015; Quartett, Inc.). Subsequently, the samples were incubated on ice for at least $1 \mathrm{~h}$ and centrifuged at $16,000 \mathrm{x}$ g for $20 \mathrm{~min}$ at $4^{\circ} \mathrm{C}$ (Smart R17 Plus Micro Centrifuge; Hanil Scientific, Inc.). Following protein concentration estimation of the supernatants using the BCA assay, the proteins were denatured at $95^{\circ} \mathrm{C}$ for $5 \mathrm{~min}$ in a 5X SDS-PAGE loading buffer (cat. no. EBA-1052; Elpis Biotech, Inc.) and separated on a $10 \%$ SDS-PAGE gel. The protein bands were transferred onto PVDF membranes (Immobilon-P Transfer membrane; MilliporeSigma); the membranes were then immersed in a blocking solution [1\% bovine serum albumin (cat. no. A0100-010; Gendepot, Inc.) in Tris-buffered saline with $0.1 \%$ Tween-20] for $1 \mathrm{~h}$ at room temperature. Next, the membranes were incubated overnight at $4^{\circ} \mathrm{C}$ with primary antibodies against inhibin $\beta$-A (INHBA), homeobox A2 (HOXA2), potassium voltage-gated channel subfamily A regulatory beta subunit 3 (KCNAB3), CTGF, and $\beta$-actin, at the following dilutions: Mouse INHBA (1:200, cat. no. sc-166503; Santa Cruz Biotechnology, Inc.), rabbit HOXA2 (1:1,000, cat. no. PA568986; Invitrogen; Thermo Fisher Scientific, Inc.), rabbit KCNAB3 (1:1,000, cat. no. AV35151; Sigma-Aldrich; Merck KGaA), mouse CTGF 

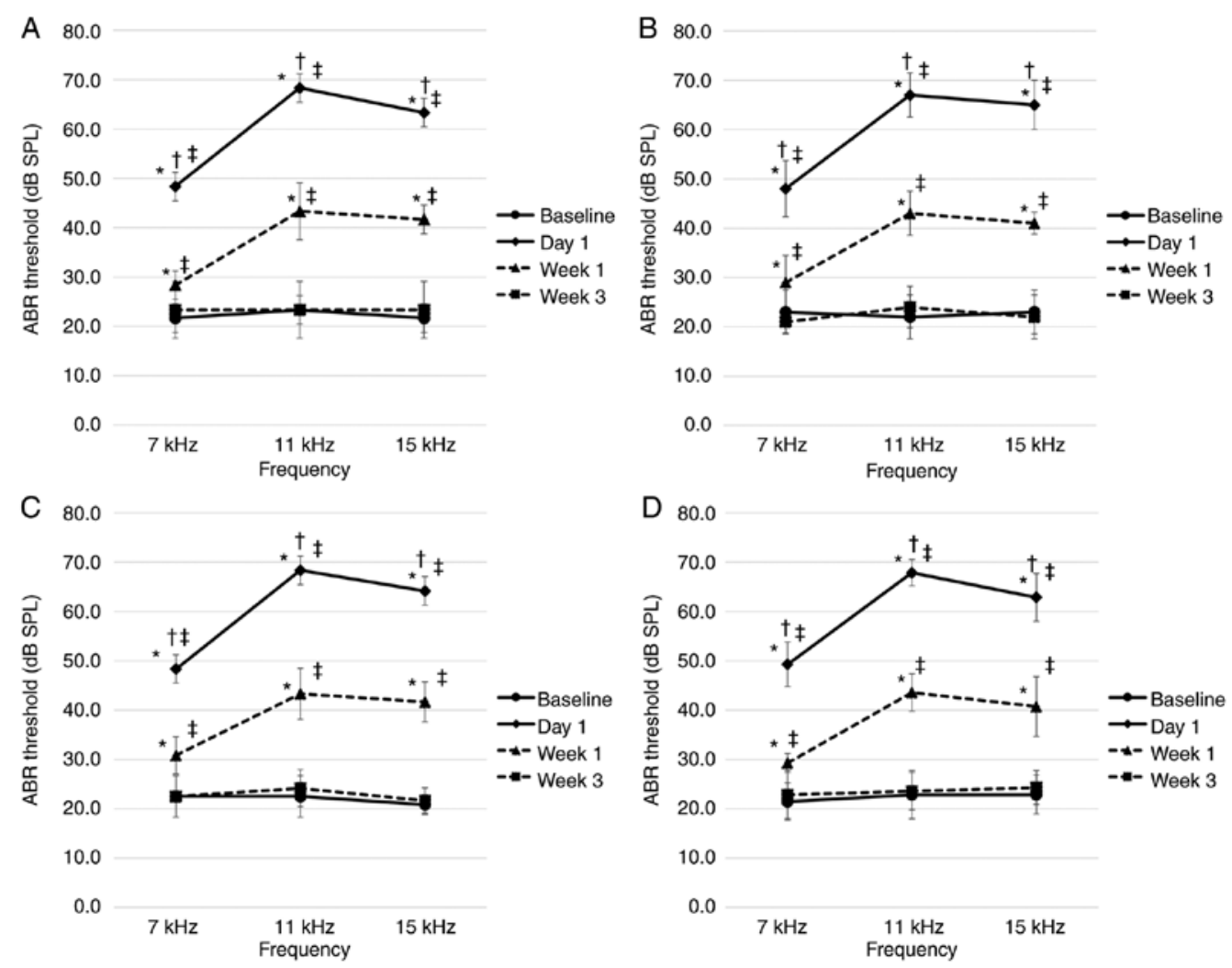

Figure 2. ABR thresholds before and after noise exposure in the first and second stages. Stage one: (A) Non-tinnitus group (n=3) and (B) tinnitus group (n=5). Stage two: (C) Non-tinnitus group $(n=6)$ and (D) tinnitus group $(n=7)$. There were no significant differences in the ABR thresholds of the rats in the tinnitus and non-tinnitus groups at all time points tested. Data are presented as mean \pm standard deviation, as determined using two-way repeated measures $A N O V A$. ${ }^{2}<0.05$ compared with baseline; ${ }^{\dagger} \mathrm{P}<0.05$ compared with week 1 ; and ${ }^{\ddagger} \mathrm{P}<0.05$ compared with week 3 . ABR, auditory brainstem response; SPL, sound pressure level.

(1:1,000, cat. no. sc-101586; Santa Cruz Biotechnology, Inc.), and mouse $\beta$-actin $(1: 3,000$, cat. no. sc-47778; Santa Cruz Biotechnology, Inc.). On the following day, the membranes were rinsed and incubated with species-specific horseradish peroxidase-conjugated secondary antibodies (anti-rabbit: 1:10,000, cat. no. ADI-SAB-300-J; and anti-mouse: 1:10,000, cat. no. ADI-SAB-100-J; both from Enzo Life Sciences, Inc.) for $1 \mathrm{~h}$ at room temperature. The protein bands were visualized using ECL solution (cat. no. WBKLS0500; Immobilon Western Chemiluminescent HRP Substrate; MilliporeSigma) and analyzed with a chemiluminescence image analyzer (ChemiDoc; Bio-Rad Laboratories, Inc.).

Statistical analysis. Statistical analysis was performed using the IBM SPSS 21.0 software (IBM Corp.). The ABR thresholds were assessed using the two-way repeated measures ANOVA followed by Tukey's post hoc test. The RQ values obtained from the RT-qPCR and western blot analyses results were examined using the Mann-Whitney U-test. The effect of miR-375-3p overexpression was analyzed using the Pearson's $\chi^{2}$ test. $\mathrm{P}<0.05$ indicated statistically significant differences.

\section{Results}

$A B R$ recordings. Prior to noise exposure, the ABR thresholds on both the right and left sides of all rats ranged between 20 and $30 \mathrm{~dB}$ SPL, at all frequencies, with no significant difference detected between the tinnitus and non-tinnitus groups at all stages. In all groups and at all frequencies, the ABR thresholds on the right side on day 1 after noise exposure were significantly higher compared with those at all other time points. After 1 week of noise exposure, the ABR threshold was significantly lower compared with that on day 1 , but significantly higher compared with the baseline threshold and the threshold 3 weeks later. However, there was no significant difference between the baseline ABR threshold and the ABR threshold after 3 weeks of exposure to noise. The ABR thresholds on the left side ranged between 20 and $30 \mathrm{~dB}$ SPL. At all time points post-exposure, the measured ABR thresholds at each frequency did not differ significantly between the two groups at the first and second stages during the experiments (Fig. 2). In addition, there were no significant differences in the ABR thresholds among the four experimental groups in the third stage (Fig. 3).

Behavioral test for tinnitus. Among individual rats, the pre-exposure $\mathrm{G} / \mathrm{N}$ ratios ranged from 30 to $70 \%$. Compared with the no-gap condition, all rats showed significant decreases in startle responses under the gap condition at all frequencies $(\mathrm{P}<0.05)$. As stated previously, the GPIAS responses were recorded at 3 weeks following noise exposure. In the first stage of the experiment, 5 of the 8 rats examined exhibited no significant decrease in the startle response under the gap condition at one or more frequencies $(\mathrm{P}>0.05)$. Consequently, these rats manifested behavioral evidence of tinnitus. The remaining rats exhibited significant decreases in startle responses under the gap condition at all frequencies; hence, they manifested no behavioral evidence of tinnitus. In the second stage of the experiment, 7 of the 13 rats examined exhibited no significant 

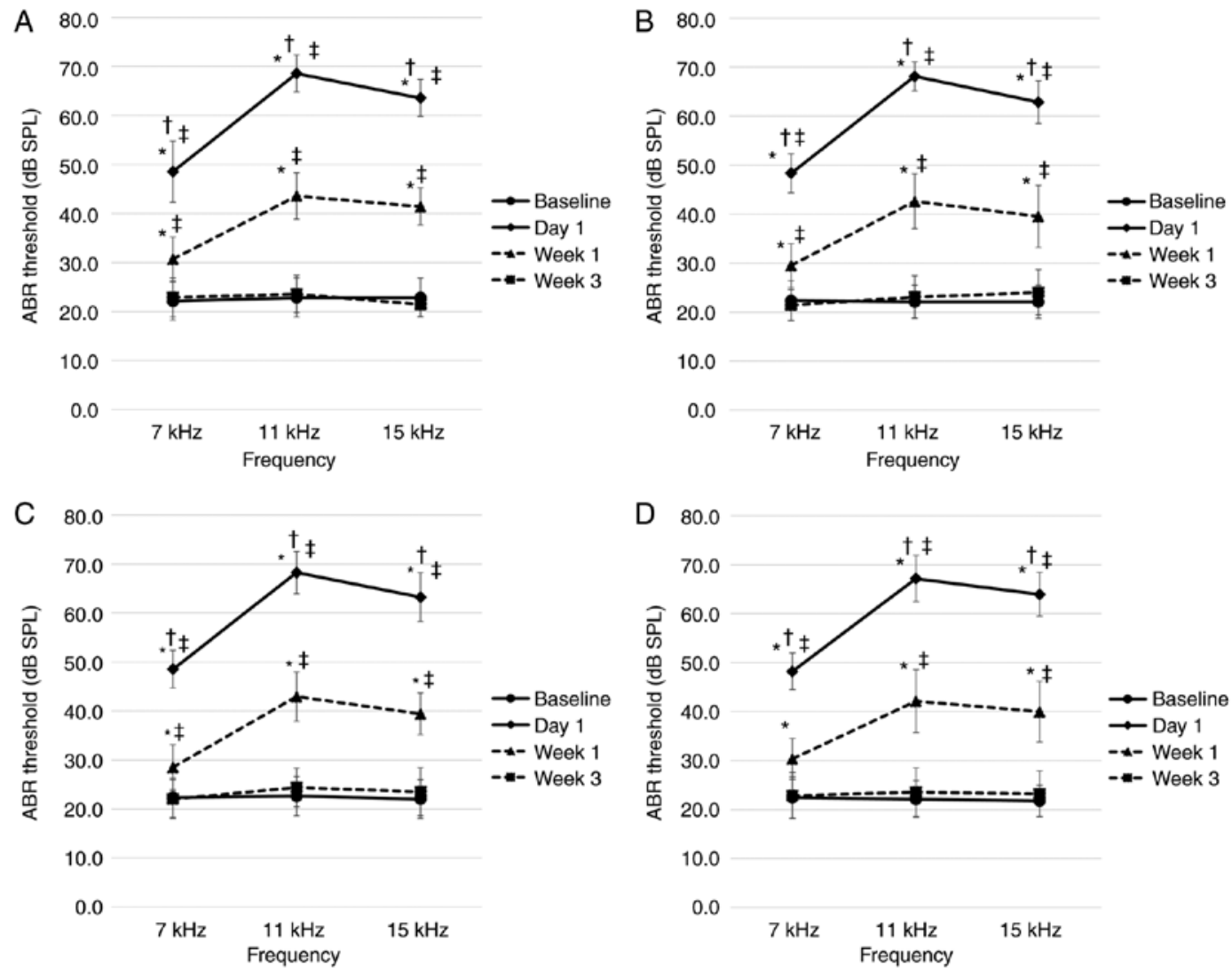

Figure 3. ABR thresholds before and after noise exposure in the third stage. (A) Non-tinnitus (n=7) and (B) tinnitus (n=21) groups with miR-375-3p mimic negative control injection. (C) Non-tinnitus ( $n=17)$ and (D) tinnitus $(n=14)$ groups with miR-375-3p mimic injection. There were no significant differences in the ABR thresholds among the rats in all groups at all time points tested. Data are presented as mean \pm standard deviation, as determined using two-way repeated measures ANOVA. ${ }^{*} \mathrm{P}<0.05$ compared with baseline, ${ }^{\dagger} \mathrm{P}<0.05$ compared with week 1 and ${ }^{\dagger} \mathrm{P}<0.05$ compared with week 3 . ABR, auditory brainstem response; SPL, sound pressure level.

decrease in startle response under the gap condition at one or more frequencies $(\mathrm{P}>0.05)$. Thus, these rats manifested behavioral evidence of tinnitus. The remaining rats exhibited significant decreases in startle responses under the gap condition at all frequencies, and were accordingly considered to manifest no behavioral evidence of tinnitus.

Selection of candidate miRNAs based on microarray analysis. Candidate miRNAs were selected based on the results obtained from microarray analysis (non-tinnitus group, $n=3$; tinnitus group, $n=3$ ). First, miRNAs not expressed in humans were excluded. Subsequently, the remaining miRNAs that satisfied the following criterion were selected: Log-ratio intensity $>0.379$ or $<-0.379(\mathrm{P}<0.08)$ between the tinnitus and non-tinnitus groups, as determined by the Student's t-test. Using this criterion, miR-15b-3p, -105, -221-3p, -375-3p, $-455-5 p,-544-5 p,-708-5 p$ and -759 were selected as candidate miRNAs (Tables I and SI).

Validation of miRNA expression using RT-qPCR. To validate the candidate miRNAs, RT-qPCR was performed (non-tinnitus group, $\mathrm{n}=6$; tinnitus group, $\mathrm{n}=7$ ) to identify those miRNAs showing a significant difference in RQ values in the tinnitus and non-tinnitus groups. It was observed that, among all the candidate miRNAs, the RQ value of miR-375-3p was significantly decreased in the tinnitus group compared with that in the non-tinnitus group $(\mathrm{P}=0.028)$, while the $\mathrm{RQ}$ values
Table I. Candidate microRNAs selected based on microarray analysis.

\begin{tabular}{lll}
\hline Candidate microRNAs & Log-ratio & P-value
\end{tabular}

Log-ratio between the tinnitus and non-tinnitus groups:

$>0.379(\mathrm{P}<0.08)$

$\begin{array}{lll}\operatorname{miR}-15 b-3 p & 0.456 & 0.020 \\ \operatorname{miR}-221-3 p & 0.500 & 0.033 \\ \operatorname{miR}-455-5 p & 0.394 & 0.076 \\ \operatorname{miR}-544-5 p & 0.666 & 0.012 \\ \operatorname{miR}-708-5 p & 0.773 & 0.043\end{array}$

Log-ratio between the tinnitus and non-tinnitus groups:

$<-0.379(\mathrm{P}<0.08)$

$\begin{array}{lll}\text { miR-105 } & -0.519 & 0.030 \\ \operatorname{miR}-375-3 p & -0.530 & 0.059 \\ \operatorname{miR}-759 & -0.661 & 0.058\end{array}$

miR, microRNA.

of miR-15b-3p, -105, -455-5p, -544-5p and -708-5p were not significantly different (Fig. 4). The Cq value of miR-759 was $>35$ even with an increased amount of total RNA as template. As this same observation was made in multiple replicates of 


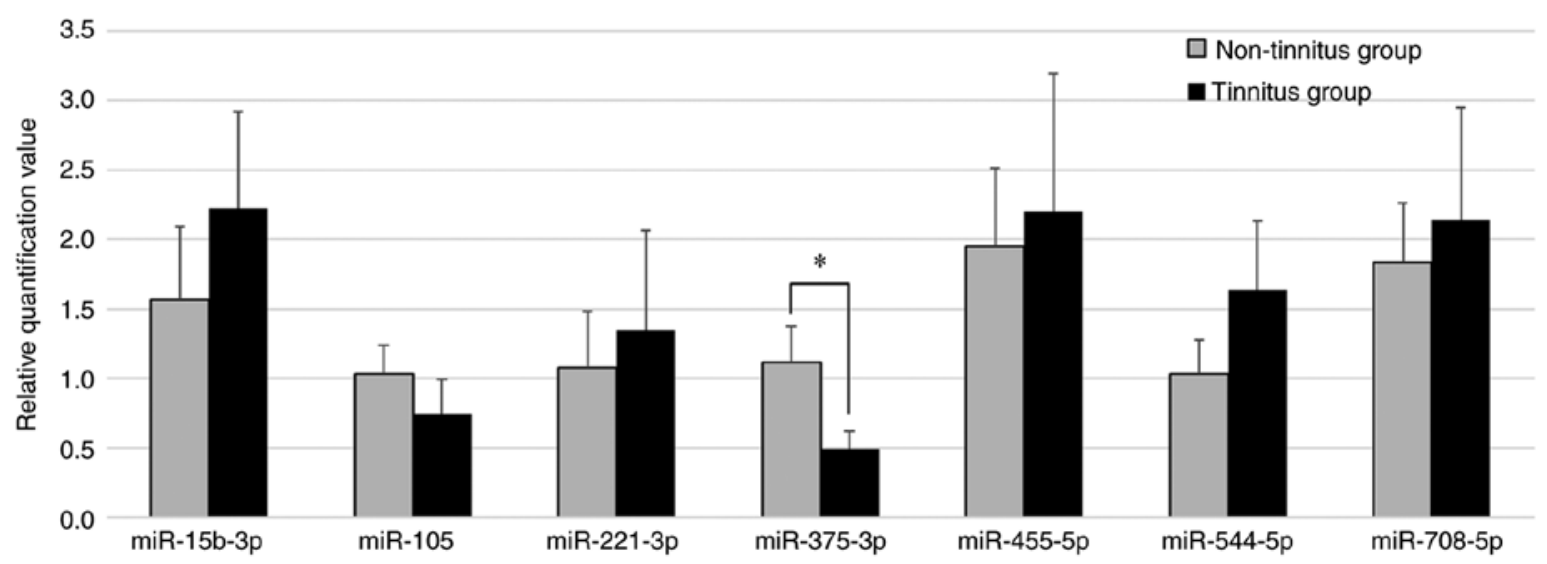

Figure 4. Reverse transcription-quantitative PCR of candidate miRNAs. The RQ value of miR-375-3p was significantly decreased in the tinnitus group $(n=7)$ compared with that in the non-tinnitus group $(n=6)$. The experiment was repeated thrice. * $\mathrm{P}<0.05$ vs. non-tinnitus group, as determined using the Mann-Whitney test. The bars indicate standard error. RQ, relative quantification; miRNA/miR, microRNA.

the experiment, miR-759 was excluded from the candidate miRNAs. Consequently, it was inferred that miR-375-3p was involved in the development of tinnitus.

Injection of miR-375-3p oligomers. To evaluate the role of miR-375-3p in the development of tinnitus, a miR-375-3p mimic or a mimic negative control was injected into the lateral ventricles of rats with tinnitus at 1 week post-noise exposure. Two weeks later (i.e., 3 weeks post-noise exposure), GPIAS recordings were performed to determine whether tinnitus persisted. It was found that tinnitus persisted in 21 of the 28 rats $(75.0 \%)$ injected with the miR-375-3p mimic negative control, and in 14 of the 31 rats $(45.2 \%)$ injected with the miR-375-3p mimic. A total of 17 rats (54.8\%) exhibited no tinnitus at 3 weeks post-noise exposure (Fig. 5 and Table II). The effect of miR-375-3p mimic on preventing the persistence of tinnitus at 3 weeks post-noise exposure was found to be statistically significant $[\mathrm{P}=0.020$, odds ratio $(\mathrm{OR})=0.275,95 \%$ confidence interval (CI): 0.090-0.833]. These results suggested that a decrease in miR-375-3p level may play a key role in the persistence of tinnitus.

Target prediction for $m i R-375-3 p$. To discern the probable targets regulated by miR-375-3p, mRNA microarray analysis was performed (Table SII). In the mimic non-tinnitus group $(n=3)$, tinnitus had ceased after miR-375-3p mimic injection, whereas in the control tinnitus group $(n=3)$, the rats had persistent tinnitus after miR-375-3p mimic negative control injection. The target gene candidates that exhibited decreased expression in the mimic non-tinnitus group were selected using the following criteria: Log-ratio $<-0.585$ and $\mathrm{P} \leq 0.25$. As an additional process for target prediction, the miRNA target prediction program TargetScan was employed (http://www.targetscan.org/cgi-bin/targetscan/vert_72/targetscan. cgi?species $=$ Rat $\&$ gid $=\&$ mir_sc $=$ miR-375-3p\&mir_c $=\&$ mir $\mathrm{nc}=\&$ sortType $=\mathrm{cs} \&$ allTxs $=\&$ incl_nc $=$ All). Genes that were predicted as targets for miR-375-3p and whose target site nucleotide sequences in rats were identical to or contained one nucleotide difference when compared with the respective genes in humans were selected from TargetScan. Collectively, the results from mRNA microarray analysis and
TargetScan revealed three gene targets: $K C N A B 3, I N H B A$ and HOXA2 (Table III).

Along with these genes, $C T G F$, a previously reported target of miR-375-3p (23), was included, and western blotting was performed to analyze the expression of these potential genes in the mimic non-tinnitus $(n=8)$ and control tinnitus $(n=7)$ experimental groups. The western blotting results showed that the mimic non-tinnitus group, wherein tinnitus ceased after 3 weeks of noise exposure owing to the injection of the mimic, exhibited a statistically significant decrease in CTGF levels compared to the control tinnitus group, in which tinnitus persisted $(\mathrm{P}=0.028)$ (Fig. 6). The other three proteins did not differ significantly between the groups.

\section{Discussion}

Although numerous studies have investigated the probable causal factors contributing to the development of tinnitus (9-11), no effective therapeutic methods have been identified to date for the treatment of this condition. To address this issue, it is necessary to identify and block the checkpoints in various pathways associated with the induction of tinnitus.

It is well known that the majority of genes are regulated by miRNAs, which are non-coding RNAs that modify gene expression via post-transcriptional regulation. Moreover, one single miRNA can regulate the expression of multiple genes, thereby serving as a checkpoint for disease outbreaks. miRNAs are particularly abundant in the brain and play important roles in the development and functioning of neuronal networks, including the regulation of neurogenesis, synaptogenesis and morphogenesis (24-26). Various neuronal diseases, including schizophrenia, autism, fragile X, Rett and Down syndromes, have also been reported to be associated with aberrant miRNA expression (27-29). In addition, vestibular compensation, a change in brainstem function concomitant with a decrease in sensory input, has been reported to be regulated by miRNAs (21). Given that tinnitus is known to be induced by changes in the brainstem in response to a reduced auditory input (9-11), the developmental mechanism of tinnitus may have similarities to the vestibular compensation process. Accordingly, it was hypothesized that one or more miRNAs 
Table II. Changes in GPIAS responses in the mimic non-tinnitus group wherein tinnitus ceased following miR-375-3p mimic injection.

\begin{tabular}{|c|c|c|c|c|c|c|}
\hline \multirow[b]{3}{*}{ No. } & \multicolumn{6}{|c|}{ Background noise $(\mathrm{kHz})$} \\
\hline & \multicolumn{2}{|c|}{$6-8$} & \multicolumn{2}{|c|}{$10-12$} & \multicolumn{2}{|c|}{$14-16$} \\
\hline & $\mathrm{G} / \mathrm{N}$ ratio $^{\mathrm{a}}$ & P-value & $\mathrm{G} / \mathrm{N}$ ratio $^{\mathrm{a}}$ & P-value & $\mathrm{G} / \mathrm{N}$ ratio $^{\mathrm{a}}$ & P-value \\
\hline \multicolumn{7}{|l|}{1} \\
\hline Baseline & 0.567 & 0.002 & 0.331 & $<0.001$ & 0.284 & $<0.001$ \\
\hline Week 1 & 1.100 & 0.793 & 0.360 & 0.001 & 0.559 & 0.223 \\
\hline Week 3 & 0.599 & $<0.001$ & 0.373 & 0.001 & 0.665 & 0.024 \\
\hline \multicolumn{7}{|l|}{2} \\
\hline Baseline & 0.477 & 0.001 & 0.571 & 0.028 & 0.249 & $<0.001$ \\
\hline Week 1 & 0.980 & 0.497 & 0.782 & 0.093 & 0.323 & 0.002 \\
\hline Week 3 & 0.176 & $<0.001$ & 0.222 & 0.010 & 0.397 & 0.014 \\
\hline \multicolumn{7}{|l|}{3} \\
\hline Baseline & 0.728 & $<0.001$ & 0.771 & 0.006 & 0.701 & $<0.001$ \\
\hline Week 1 & 0.730 & $<0.001$ & 0.972 & 0.908 & 0.960 & 0.315 \\
\hline Week 3 & 0.785 & 0.006 & 0.785 & 0.003 & 0.842 & 0.015 \\
\hline \multicolumn{7}{|l|}{4} \\
\hline Baseline & 0.638 & $<0.001$ & 0.567 & 0.001 & 0.568 & 0.005 \\
\hline Week 1 & 0.984 & 0.627 & 0.744 & 0.010 & 0.972 & 0.576 \\
\hline Week 3 & 0.692 & 0.005 & 0.714 & 0.044 & 0.608 & $<0.001$ \\
\hline \multicolumn{7}{|l|}{5} \\
\hline Baseline & 0.537 & 0.001 & 0.295 & 0.001 & 0.296 & $<0.001$ \\
\hline Week 1 & 0.246 & 0.002 & 0.966 & 0.106 & 0.289 & 0.001 \\
\hline Week 3 & 0.247 & 0.002 & 0.329 & 0.003 & 0.964 & 0.020 \\
\hline \multicolumn{7}{|l|}{6} \\
\hline Baseline & 0.583 & 0.002 & 0.532 & $<0.001$ & 0.679 & 0.011 \\
\hline Week 1 & 0.970 & 0.852 & 1.031 & 0.890 & 0.623 & 0.003 \\
\hline Week 3 & 0.734 & 0.033 & 0.692 & 0.005 & 0.602 & 0.004 \\
\hline \multicolumn{7}{|l|}{7} \\
\hline Baseline & 0.749 & 0.045 & 0.368 & $<0.001$ & 0.451 & 0.001 \\
\hline Week 1 & 0.401 & $<0.001$ & 0.703 & 0.014 & 1.015 & 0.633 \\
\hline Week 3 & 0.734 & 0.015 & 0.642 & 0.026 & 0.421 & 0.001 \\
\hline \multicolumn{7}{|l|}{8} \\
\hline Baseline & 0.121 & $<0.001$ & 0.357 & $<0.001$ & 0.443 & 0.015 \\
\hline Week 1 & 1.092 & 0.663 & 0.258 & $<0.001$ & 0.148 & $<0.001$ \\
\hline Week 3 & 0.154 & $<0.001$ & 0.108 & $<0.001$ & 0.101 & $<0.001$ \\
\hline \multicolumn{7}{|l|}{9} \\
\hline Baseline & 0.332 & $<0.001$ & 0.424 & $<0.001$ & 0.266 & $<0.001$ \\
\hline Week 1 & 0.497 & $<0.001$ & 0.583 & 0.013 & 0.667 & 0.254 \\
\hline Week 3 & 0.480 & $<0.001$ & 0.354 & $<0.001$ & 0.284 & 0.013 \\
\hline \multicolumn{7}{|l|}{10} \\
\hline Baseline & 0.285 & 0.001 & 0.373 & 0.002 & 0.558 & 0.001 \\
\hline Week 1 & 0.378 & $<0.001$ & 0.765 & 0.178 & 0.990 & 0.760 \\
\hline Week 3 & 0.343 & $<0.001$ & 0.343 & 0.016 & 0.533 & 0.010 \\
\hline \multicolumn{7}{|l|}{11} \\
\hline Baseline & 0.624 & 0.006 & 0.642 & 0.029 & 0.526 & 0.001 \\
\hline Week 1 & 1.026 & 0.020 & 1.006 & 0.443 & 0.669 & 0.036 \\
\hline Week 3 & 0.378 & 0.002 & 0.564 & 0.011 & 0.327 & 0.008 \\
\hline \multicolumn{7}{|l|}{12} \\
\hline Baseline & 0.579 & 0.006 & 0.300 & $<0.001$ & 0.199 & $<0.001$ \\
\hline Week 1 & 1.077 & 0.358 & 0.858 & 0.310 & 0.519 & 0.002 \\
\hline Week 3 & 0.616 & 0.001 & 0.555 & 0.007 & 0.448 & 0.040 \\
\hline
\end{tabular}


Table II. Continued.

\begin{tabular}{|c|c|c|c|c|c|c|}
\hline \multirow[b]{3}{*}{ No. } & \multicolumn{6}{|c|}{ Background noise $(\mathrm{kHz})$} \\
\hline & \multicolumn{2}{|c|}{$6-8$} & \multicolumn{2}{|c|}{$10-12$} & \multicolumn{2}{|c|}{$14-16$} \\
\hline & $\mathrm{G} / \mathrm{N}$ ratio $^{\mathrm{a}}$ & P-value & $\mathrm{G} / \mathrm{N}$ ratio $^{\mathrm{a}}$ & P-value & $\mathrm{G} / \mathrm{N}$ ratio $^{\mathrm{a}}$ & P-value \\
\hline \multicolumn{7}{|l|}{13} \\
\hline Baseline & 0.595 & 0.001 & 0.452 & 0.002 & 0.358 & 0.001 \\
\hline Week 1 & 0.589 & 0.001 & 0.561 & 0.012 & 0.812 & 0.101 \\
\hline Week 3 & 0.479 & $<0.001$ & 0.723 & 0.036 & 0.514 & 0.001 \\
\hline \multicolumn{7}{|l|}{14} \\
\hline Baseline & 0.453 & $<0.001$ & 0.669 & 0.038 & 0.668 & 0.012 \\
\hline Week 1 & 0.440 & $<0.001$ & 0.623 & 0.004 & 0.770 & 0.165 \\
\hline Week 3 & 0.421 & 0.007 & 0.345 & $<0.001$ & 0.498 & 0.001 \\
\hline \multicolumn{7}{|l|}{15} \\
\hline Baseline & 0.455 & 0.001 & 0.507 & 0.024 & 0.379 & 0.001 \\
\hline Week 1 & 1.311 & 0.102 & 0.705 & 0.254 & 0.310 & 0.004 \\
\hline Week 3 & 0.518 & 0.024 & 0.502 & 0.021 & 0.433 & 0.025 \\
\hline \multicolumn{7}{|l|}{16} \\
\hline Baseline & 0.560 & 0.017 & 0.620 & 0.014 & 0.312 & $<0.001$ \\
\hline Week 1 & 0.286 & 0.047 & 0.596 & 0.141 & 0.532 & 0.006 \\
\hline Week 3 & 0.511 & $<0.001$ & 0.362 & $<0.001$ & 0.483 & 0.002 \\
\hline \multicolumn{7}{|l|}{17} \\
\hline Baseline & 0.594 & 0.005 & 0.486 & $<0.001$ & 0.611 & 0.021 \\
\hline Week 1 & 1.061 & 0.818 & 0.727 & 0.044 & 0.618 & 0.036 \\
\hline Week 3 & 0.467 & $<0.001$ & 0.650 & 0.040 & 0.741 & 0.049 \\
\hline
\end{tabular}

${ }^{a}$ RMS-GSR/RMS-NGSR. RMS, root-mean-square; GSR, gap-conditioned startle response; NGSR, non-gap-conditioned startle response; GPIAS, gap pre-pulse inhibition of acoustic startle reflex.

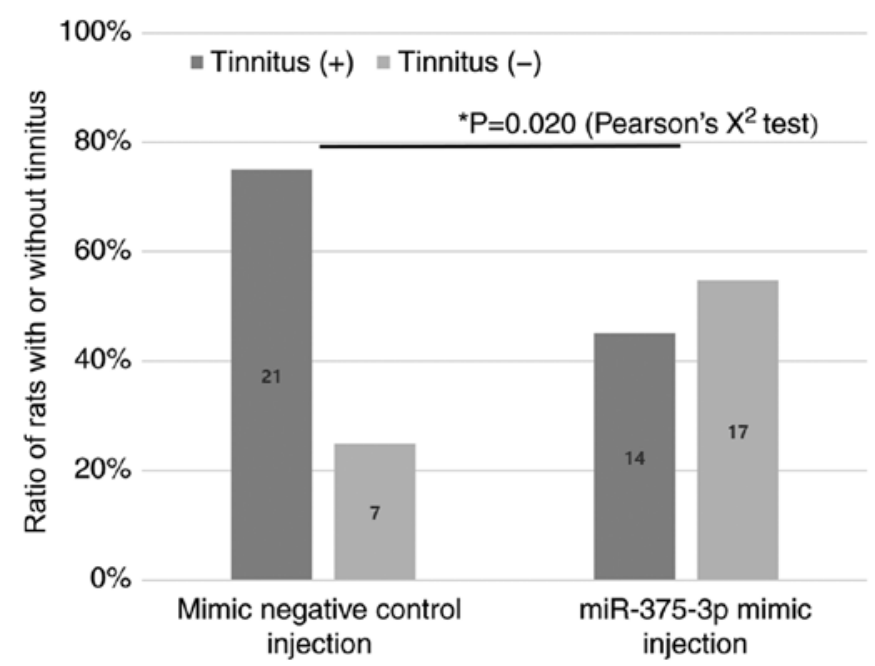

Figure 5. Ratio of rats with or without tinnitus post-injection. miR-375-3p mimic and miR-375-3p mimic negative control were administered to 31 and 28 rats, respectively, that exhibited evidence of tinnitus at 1 week post-noise exposure. Two weeks later (i.e., 3 weeks after noise exposure), the GPIAS responses were measured to determine whether tinnitus persisted. The mimic-injected group exhibited a significantly lower ratio of animals with persistent tinnitus at 3 weeks post-noise exposure, as determined using the Pearson's $\chi^{2}$ test $(\mathrm{P}=0.020$, odds ratio $=0.275,95 \%$ confidence interval: $0.090-0.833)$. The number of animals in each experimental group are labeled in the graph. GPIAS, gap pre-pulse inhibition of acoustic startle reflex; miR, microRNA. may play checkpoint roles in the development of tinnitus and, thus, we sought to identify these miRNAs.

In our previous study, a tinnitus animal model was developed to elucidate the mechanisms underlying tinnitus resulting from noise exposure and inducing TTS in rats (12). Using this model, the DCNs of rats were collected and the auditory and non-auditory projections were compared between the tinnitus and the non-tinnitus groups. It was observed that a decrease in the auditory projections and a subsequent increase in the non-auditory projections via axonal sprouting were important phenomena associated with the development of tinnitus at 3 weeks post-noise exposure. In the present study, the same model was used in an effort to identify the putative miRNAs implicated in the regulation of the aforementioned processes. At 3 weeks post-noise exposure, the DCNs of the rats were collected to identify miRNAs exhibiting differential expression in the tinnitus and non-tinnitus groups and the candidate miRNAs were selected based on microarray analysis. Through subsequent validation using RT-qPCR, it was found that miR-375-3p was significantly decreased in the tinnitus group compared with the non-tinnitus group. miRNAs associated with tinnitus were identified by comparing the groups with and without tinnitus, although the same degree of hearing loss was induced in both groups. If the control group without noise exposure was also included in the comparisons, the results 

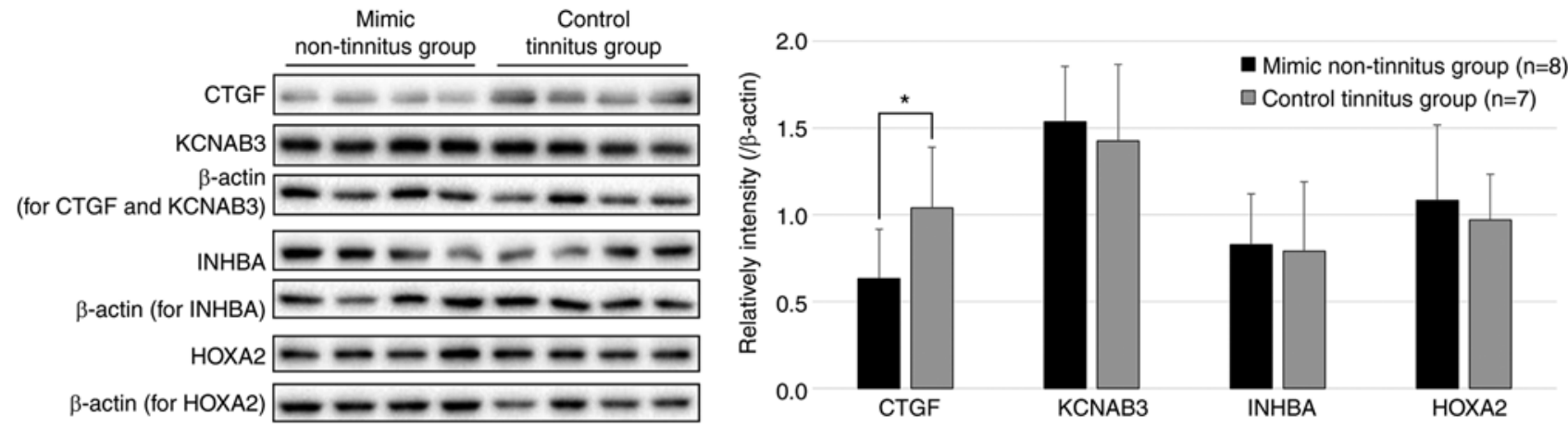

Figure 6. Representative western blots and quantitative analyses of CTGF, INHBA, HOXA2, and KCNAB3 expression levels. CTGF level was significantly lower in the mimic non-tinnitus group compared with that in the control tinnitus group $(\mathrm{P}=0.028)$. Data are presented as mean \pm standard error, as determined using the Mann-Whitney test. " $\mathrm{P}<0.05$. CTGF, connective tissue growth factor; INHBA, inhibin $\beta$-A; HOXA2, homeobox A2; KCNAB3, potassium voltage-gated channel subfamily A regulatory beta subunit 3 .

Table III. Candidate target genes of microRNA-375-3p selected based on microarray analysis.

\begin{tabular}{lcc}
\hline $\begin{array}{l}\text { Candidate } \\
\text { target genes }\end{array}$ & $\begin{array}{c}\text { Log-ratio between the mimic } \\
\text { non-tinnitus and control tinnitus } \\
\text { groups: }<-0.585(\mathrm{P}<0.25)\end{array}$ & P-value \\
\hline KCNAB3 & -0.650 & 0.136 \\
INHBA & -0.709 & 0.166 \\
HOXA2 & -0.957 & 0.240 \\
\hline
\end{tabular}

KCNAB3, potassium voltage-gated channel subfamily A regulatory beta subunit 3; INHBA, inhibin $\beta$-A; HOXA2, homeobox A2.

would have been clearer. However, even if the same degree of hearing loss occurs due to exposure to the same noise frequencies, tinnitus only occurs in a proportion of the cases. Therefore, when conducting tinnitus research, it is essential to distinguish between the changes occurring owing to hearing loss and the changes that cause tinnitus; this may be achieved by comparing tinnitus and non-tinnitus animal models. The effect of miR-375-3p on tinnitus development was evaluated by injecting a miR-375-3p mimic or a mimic negative control. In the mimic negative control-injected group, tinnitus persisted in $75.0 \%$ of the rats. In our previous study using the same tinnitus animal model, we found that tinnitus persisted 3 weeks after noise exposure in $81.5 \%$ of animals that developed tinnitus at 1 week after noise exposure (12). Similar results were also obtained in the present study. However, in the mimic-injected group, the proportion of animals with persistent tinnitus decreased significantly to $45.2 \%$. This finding confirmed that the overexpression of miR-375-3p could reduce the persistence of tinnitus.

Several researchers have reported the effects of miR-375 on the brain, although it is found in multiple organs or tissues (30-32). For example, the expression of miR-375 was found to be downregulated in several neural injury models, including models of cerebral ischemia/reperfusion injury (33-35). However, the overexpression of miR-375, using an miR-375 mimic, has been shown to provide significant protection against the ischemia/reperfusion-induced brain injury by reducing cell apoptosis. A recent study reported that $C T G F$ is one of the targets of miR-375-3p, and that the overexpression of miR-375-3p resulted in the downregulation of CTGF in a brain ischemia/reperfusion injury model (23). CTGF has been demonstrated to exert pro-apoptotic effects under different conditions, including carcinoma and brain injury. CTGF levels have been demonstrated to increase in the brains of rats with traumatic brain injury (36). The CTGF protein has been shown to have pro-apoptotic activity, and to promote a reduction in neuronal survival $(37,38)$.

Our previous study demonstrated that, subsequent to hearing loss, the auditory projections degrade quickly and more severely in the tinnitus group compared with the non-tinnitus group, thereby leading to a significant increase in the somatosensory projections. This has been identified as an important process in the development of tinnitus (12). In the present study, it was observed that miR-375-3p expression decreased in the tinnitus group compared with the non-tinnitus group. Considering the changes in miR-375-3p expression following neural injury, it was hypothesized that the development of tinnitus may be attributed to the decreased expression of miR-375-3p. In addition, when miR-375-3p was overexpressed with miR-375-3p mimic injection, the proportion of animals with sustained tinnitus to those with ceased tinnitus decreased significantly compared with the control group. It was inferred that miR-375-3p may prevent the persistence of tinnitus by attenuating the neural damage following noise exposure. This mechanism may be involved in the apoptotic process of $C T G F$, one of the gene targets of miR-375-3p. A possible scenario is that the downregulation of CTGF by miR-375-3p is weakened as the expression of miR-375-3p decreases, leading to more severe neural damage due to increased CTGF expression and the apoptosis of auditory neurons. Considering that there was no difference in the hearing level of the rats in the tinnitus and non-tinnitus groups, it was hypothesized that there may be differences in the degree of damage of high-threshold fibers, rather than the low-threshold fibers, which determine the hearing thresholds (39). On the other hand, it was observed that tinnitus ceased in some animals administered the mimic negative control. This is consistent with our previous report, which demonstrated that $18.5 \%$ of animals exhibiting evidence of tinnitus at 1 week post-noise exposure exhibited no tinnitus at 3 weeks post-noise exposure, even though no specific 
treatment was administered, under identical experimental settings (12). This suggests that the susceptibility of auditory neurons to damage by noise exposure varies among individuals, and that the individual differences in miR-375-3p expression may be responsible for this varied susceptibility. In addition, some animals exhibited persistent tinnitus even after the administration of miR-375-3p mimic. This suggests that other factors, besides miR-375-3p expression, may also be involved in tinnitus development. This discrepancy may also be due to certain changes in other pathway(s) that cannot be compensated or reversed by miR-375-3p supplementation, such as the limbic system, which is associated with the occurrence and persistence of tinnitus (40). However, further research is necessary to verify this hypothesis.

There were certain limitations to the present study that must be acknowledged. Only male rats were selected in this study. The rats were ensured to be an identical strain and within a certain age range to reduce variabilities. Furthermore, ABR recordings were checked before noise exposure to prove that the hearing did not differ between the groups. Interestingly, female rats do not display greater variability during the reproductive cycle compared with male rats; furthermore, male and female mice and humans also have similar levels of variability in terms of gene expression (41-43). Therefore, it is inferred that using both sexes in this study would not have made a significant difference in the results, provided no difference in the hearing levels was confirmed between the tinnitus and non-tinnitus groups. However, for the results of this experiment to be translated into treatment strategies in the future, additional studies using female rats may be helpful.

To the best of our knowledge, no previous studies have examined the involvement of miRNAs in the development of tinnitus. In the present study, the expression of miR-375-3p was found to be reduced in the DCNs of rats with tinnitus, and the overexpression of miR-375-3p prevented the persistence of tinnitus by reducing the expression of $C T G F$. These findings will contribute significantly to the development of a novel therapeutic approach to tinnitus, thereby bringing about a significant breakthrough in the treatment of this potentially debilitating condition.

\section{Acknowledgements}

Not applicable.

\section{Funding}

The present study was supported by the National Research Foundation of Korea funded by the Korea government Ministry of Education (grant no. 2018R1D1A1A02085478) and the Chung-Ang University Research Grants provided in 2020 .

\section{Availability of data and materials}

The raw data of microRNA microarray analysis generated during the current study are available in the Gene Expression Omnibus (GEO) (accession numbers: GSE172259), [https://www.ncbi. nlm.nih.gov/geo/query/acc.cgi?acc=GSE172259].

\section{Authors' contributions}

MC designed the experiments and study. $\mathrm{KHH}, \mathrm{HC}, \mathrm{KRH}$ and MC performed the experiments. $\mathrm{MC}, \mathrm{KHH}$ and SKM have seen and confirm the authenticity of the raw data. MC and $\mathrm{KHH}$ wrote the manuscript, analyzed and interpreted the data. SKM, YKK and IP contributed to designing the experiment and revising the manuscript. All the authors have read and approved the final manuscript.

\section{Ethics approval and consent to participate}

All procedures for animal care and use were approved by the Institutional Animal Care and Use Committee of Chung-Ang University (2016-00092).

\section{Patient consent for publication}

Not applicable.

\section{Competing interests}

The authors declare that they have no competing interests.

\section{References}

1. Axelsson A and Ringdahl A: Tinnitus-a study of its prevalence and characteristics. Br J Audiol 23: 53-62, 1989.

2. Hébert S, Canlon B and Hasson D: Emotional exhaustion as a predictor of tinnitus. Psychother Psychosom 81: 324-326, 2012.

3. Langguth B: A review of tinnitus symptoms beyond 'ringing in the ears': A call to action. Curr Med Res Opin 27: 1635-1643, 2011.

4. Shargorodsky J, Curhan GC and Farwell WR: Prevalence and characteristics of tinnitus among US adults. Am J Med 123: 711-718, 2010.

5. Jastreboff PJ: Tinnitus retraining therapy. Prog Brain Res 166: 415-423, 2007.

6. Moller AR: Tinnitus: Presence and future. Prog Brain Res 166: 3-16, 2007.

7. Cima RFF, Mazurek B, Haider H, Kikidis D, Lapira A, Norena A and Hoare DJ: A multidisciplinary European guideline for tinnitus: Diagnostics, assessment, and treatment. HNO 67 (Suppl 1): S10-S42, 2019.

8. TunkelDE,Bauer CA, Sun GH, Rosenfeld RM, ChandrasekharSS, Cunningham ER Jr, Archer SM, Blakley BW, Carter JM, Granieri EC, et al: Clinical practice guideline: Tinnitus. Otolaryngol Head Neck Surg 151 (Suppl 2): S1-S40, 2014.

9. Dehmel S, Pradhan S, Koehler S, Bledsoe S and Shore S: Noise overexposure alters long-term somatosensory-auditory processing in the dorsal cochlear nucleus-possible basis for tinnitus-related hyperactivity? J Neurosci 32: 1660-1671, 2012.

10. Koehler SD and Shore SE: Stimulus timing-dependent plasticity in dorsal cochlear nucleus is altered in tinnitus. J Neurosci 33: 19647-19656, 2013.

11. Zeng C, Yang Z, Shreve L, Bledsoe S and Shore S: Somatosensory projections to cochlear nucleus are upregulated after unilateral deafness. J Neurosci 32: 15791-15801, 2012.

12. Han KH, Mun SK, Sohn S, Piao XY, Park I and Chang M: Axonal sprouting in the dorsal cochlear nucleus affects gap-prepulse inhibition following noise exposure. Int J Mol Med 44: 1473-1483, 2019.

13. Hollins SL and Cairns MJ: MicroRNA: Small RNA mediators of the brains genomic response to environmental stress. Prog Neurobiol 143: 61-81, 2016.

14. Campbell $\mathrm{K}$ and Booth SA: MicroRNA in neurodegenerative drug discovery: The way forward? Expert Opin Drug Discov 10: 9-16, 2015.

15. Esteller M: Non-coding RNAs in human disease. Nat Rev Genet 12: 861-874, 2011. 
16. Gebert LF, Rebhan MA, Crivelli SE, Denzler R, Stoffel M and Hall J: Miravirsen (SPC3649) can inhibit the biogenesis of miR-122. Nucleic Acids Res 42: 609-621, 2014.

17. National Research Council: Guide for the Care and Use of Laboratory Animals. The National Academies Press, Inc., Washington, DC, 2011.

18. Paxinos $G$ and Watson $C$ : The Rat Brain in Stereotaxic Coordinates. Academic Press, Inc., Burlington, MA, 2006.

19. Mun SK, Han KH, Baek JT, Ahn SW, Cho HS and Chang MY: Losartan prevents maladaptive Auditory-Somatosensory plasticity after hearing loss via transforming growth Factor- $\beta$ signaling suppression. Clin Exp Otorhinolaryngol 12: 33-39, 2019.

20. Longenecker RJ and Galazyuk AV: Methodological optimization of tinnitus assessment using prepulse inhibition of the acoustic startle reflex. Brain Res 1485: 54-62, 2012.

21. Chang MY, Park S, Choi JJ, Kim YK, Suh MW, Lee JH, Oh SH and Park MK: MicroRNAs 218a-5p, 219a-5p, and 221-3p regulate vestibular compensation. Sci Rep 7: 8701, 2017.

22. Livak KJ and Schmittgen TD: Analysis of relative gene expression data using real-time quantitative PCR and the 2(-Delta Delta C(T)) Method. Methods 25: 402-408, 2001.

23. Ou J, Kou L, Liang L and Tang C: MiR-375 attenuates injury of cerebral ischemia/reperfusion via targetting Ctgf. Biosci Rep 37: BSR20171242, 2017.

24. Dugas JC, Cuellar TL, Scholze A, Ason B, Ibrahim A, Emery B, Zamanian JL, Foo LC, McManus MT and Barres BA: Dicerl and miR-219 Are required for normal oligodendrocyte differentiation and myelination. Neuron 65: 597-611, 2010.

25. Giraldez AJ, Cinalli RM, Glasner ME, Enright AJ, Thomson JM, Baskerville S, Hammond SM, Bartel DP and Schier AF: MicroRNAs regulate brain morphogenesis in zebrafish. Science 308: 833-838, 2005.

26. Schratt GM, Tuebing F, Nigh EA, Kane CG, Sabatini ME, Kiebler $\mathrm{M}$ and Greenberg ME: A brain-specific microRNA regulates dendritic spine development. Nature 439: 283-289, 2006.

27. Banerjee-Basu S, Larsen E and Muend S: Common microRNAs target established ASD genes. Front Neurol 5: 205, 2014.

28. Im HI and Kenny PJ: MicroRNAs in neuronal function and dysfunction. Trends Neurosci 35: 325-334, 2012.

29. Sun E and Shi Y: MicroRNAs: Small molecules with big roles in neurodevelopment and diseases. Exp Neurol 268: 46-53, 2015.

30. Ding L, Xu Y, Zhang W, Deng Y, Si M, Du Y, Yao H, Liu X, Ke Y, Si J and Zhou T: MiR-375 frequently downregulated in gastric cancer inhibits cell proliferation by targeting JAK2. Cell Res 20: 784-793, 2010.

31. He XX, Chang Y, Meng FY, Wang MY, Xie QH, Tang F, Li PY, Song YH and Lin JS: MicroRNA-375 targets AEG-1 in hepatocellular carcinoma and suppresses liver cancer cell growth in vitro and in vivo. Oncogene 31: 3357-3369, 2012.
32. Kapsimali M, Kloosterman WP, de Bruijn E, Rosa F, Plasterk RH and Wilson SW: MicroRNAs show a wide diversity of expression profiles in the developing and mature central nervous system. Genome Biol 8: R173, 2007.

33. Bhinge A, Namboori SC, Bithell A, Soldati C, Buckley NJ and Stanton LW: MiR-375 is essential for human spinal motor neuron development and may be involved in motor neuron degeneration. Stem Cells 34: 124-134, 2016.

34. Wang C, Pan Y, Cheng B, Chen J and Bai B: Identification of conserved and novel microRNAs in cerebral ischemia-reperfusion injury of rat using deep sequencing. J Mol Neurosci 54: 671-683, 2014.

35. Wang Y, Dong X, Li Z, Wang W, Tian J and Chen J: Downregulated RASD1 and upregulated miR-375 are involved in protective effects of calycosin on cerebral ischemia/reperfusion rats. J Neurol Sci 339: 144-148, 2014.

36. Hertel M, Tretter Y, Alzheimer C and Werner S: Connective tissue growth factor: A novel player in tissue reorganization after brain injury? Eur J Neurosci 12: 376-380, 2000.

37. Hall-Glenn F and Lyons KM: Roles for CCN2 in normal physiological processes. Cell Mol Life Sci 68: 3209-3217, 2011.

38. Khodosevich K, Lazarini F, von Engelhardt J, Kaneko H, Lledo PM and Monyer H: Connective tissue growth factor regulates interneuron survival and information processing in the olfactory bulb. Neuron 79: 1136-1151, 2013.

39. Bharadwaj HM, Masud S, Mehraei G, Verhulst S and Shinn-Cunningham BG: Individual differences reveal correlates of hidden hearing deficits. J Neurosci 35: 2161-2172, 2015.

40. Elgoyhen AB, Langguth $\mathrm{B}$, De Ridder D and Vanneste S: Tinnitus: Perspectives from human neuroimaging. Nat Rev Neurosci 16: 632-642, 2015.

41. Becker JB, Prendergast BJ and Liang JW: Female rats are not more variable than male rats: A meta-analysis of neuroscience studies. Biol Sex Differ 7: 34, 2016.

42. Itoh Y and Arnold AP: Are females more variable than males in gene expression? Meta-analysis of microarray datasets. Biol Sex Differ 6: 18, 2015 .

43. Lauer AM and Schrode KM: Sex bias in basic and preclinical noiseinduced hearing loss research. Noise Health 19: 207-212, 2017. 